\title{
Effect on mandibular length of juvenile hormones and regulation of soldier differentiation in the termite Reticulitermes speratus (Isoptera: Rhinotermitidae)
}

\author{
Mariko Tsuchiya, Dai Watanabe and Kiyoto MAEKAWA* \\ Graduate School of Science and Engineering, University of Toyama; Toyama 930-8555, Japan \\ (Received 18 October 2007; Accepted 31 January 2008)
}

\begin{abstract}
To understand the relationship between juvenile hormone $(\mathrm{JH})$ titers and morphogenetic changes associated with soldier differentiation of the termite Reticulitermes speratus, mandibular length (length between apex and first teeth) of presoldiers induced by various concentrations of JH III (0-320 $\mu \mathrm{g}$ per dish) was measured. Presoldiers were observed when treated with more than $20 \mu \mathrm{g} \mathrm{JH}$ III. Both left and right mandibular lengths were significantly different among differentiated presoldiers treated with different concentrations of JH III. Concentration-dependent effects on mandibular lengths were observed: higher JH III concentrations (160 and $320 \mu \mathrm{g})$ caused differentiation in presoldiers with longer mandibles, and lower concentrations $(40$ and $80 \mu \mathrm{g})$ caused differentiation in presoldiers with shorter mandibles. These results suggest that morphogenetic changes specific to soldiers are influenced by JH titers (endogenous $\mathrm{JH}+$ applied JH III) in workers. Live soldiers could inhibit presoldier differentiation in $R$. speratus workers. Interestingly, mandibular lengths of presoldiers induced by JH III were influenced by soldier presence, which could influence morphological changes, probably by regulation of JH titers in workers, as recently shown in the termite Coptotermes formosanus.
\end{abstract}

Key words: Termite; juvenile hormone III; soldier; presoldier; mandible

\section{INTRODUCTION}

Termites have a complicated caste system (Roisin, 2000), usually consisting of several castes, each of which attends to their own tasks. In lower termites, such as Reticulitermes speratus (Kolbe) (Rhinotermitidae), workers are immature individuals and are not at the end of their developmental pathway. They can differentiate into soldiers through a presoldier stage, supplementary reproductives and nymphs under appropriate conditions. For soldier differentiation, it is widely known that treatment of workers with juvenile hormone $(\mathrm{JH})$ or juvenile hormone analogs (JHA) resulted in higher soldier production (Doki et al., 1984; OkotKotber et al., 1991; Lelis and Everaerts, 1993; Ogino et al., 1993; Scharf et al., 2003). The dominant $\mathrm{JH}$ homolog in insects is JH III (Hartfelder, 2000) and recently, JH III was identified in the rhinotermitid Coptotermes formosanus (Park and
Raina, 2004).

$\mathrm{JH}$ titers vary during soldier differentiation in termites (reviewed in Hartfelder and Emlen, 2005). Increased $\mathrm{JH}$ titers appear to set the stage for development into soldiers, although $\mathrm{JH}$ titers can vary among individuals even within a caste (e.g., Park and Raina, 2005). Soldiers are normally unable to feed themselves and soldier proportions are relatively low in each termite colony (Haverty, 1977). The regulatory mechanisms of soldier differentiation are still poorly understood, but recent studies suggest that soldiers of C. formosanus regulate $\mathrm{JH}$ titers and caste differentiation in workers (Park and Raina, 2004, 2005; Mao et al., 2005). Park and Raina (2003) also suggested that physical contact is needed for soldiers to inhibit additional soldier differentiation in C. formosanus; however, it is still unclear which morphogenetic effects unique to soldiers are caused by soldier presence.

During soldier differentiation, various character-

\footnotetext{
* To whom correspondence should be addressed at: E-mail: kmaekawa@sci.u-toyama.ac.jp
} DOI: 10.1303/aez.2008.307 
istics are changed (Weesner, 1969). Especially in lower termites, mandibular lengths (apical region in particular) typically change from workers to presoldiers, and from presoldiers to soldiers (Lelis and Everaerts, 1993; Koshikawa, et al., 2002). However, because the morphogenesis of soldierspecific characteristics and $\mathrm{JH}$ titers have not been fully investigated, their influences on morphogenetic changes by soldier presence are not clear. In this study, we measured mandibular lengths of molted individuals after applying various concentrations of JH III to workers of the termite $R$. speratus. We then analyzed the effects on the mandibular lengths of newly molted individuals when soldiers inhibited presoldier differentiation. The goals of the present study are: (1) to understand the relationships between $\mathrm{JH}$ titers and morphogenetic changes associated with soldier differentiation, and (2) to understand the effects of soldier presence on morphogenetic changes during soldier differentiation.

\section{MATERIALS AND METHODS}

Termites. The specimens used in this study were taken from a colony collected around Keta Shrine in Ishikawa Prefecture, Japan on 20 February 2005. Divided logs were brought back to the laboratory and kept in a plastic case in constant darkness. All experimental insects were held in an incubator at $23^{\circ} \mathrm{C}$ in constant darkness for at least 3 days before use.

Experiments on various concentrations of $\mathbf{J H}$ III. According to the numbers of antenna segments and body size (Tsunoda et al., 1986; Takematsu, 1992), 4th and 5th stage workers, which can differentiate into presoldiers under natural conditions (Takematsu, 1992), were selected from the colony. Filter papers $(55 \mathrm{~mm}$ diameter; Advantec No. 1, Tokyo, Japan) were treated with various quantities of juvenile hormone III (Sigma-Aldrich Japan Inc., Tokyo, Japan) $(5,10,20,40,80,160$ and $320 \mu \mathrm{g})$ dissolved in $200 \mu \mathrm{l}$ acetone. Filter papers treated with only acetone and deionized water (DW) were also prepared for the control. After the acetone had evaporated, each filter paper was wetted with about $100 \mu \mathrm{l}$ DW and placed in a $65 \mathrm{~mm}$ Petri dish with 20 worker individuals. Each dose was replicated five times. All dishes were kept in an incubator at $23^{\circ} \mathrm{C}$ in constant darkness for 22 days. If we found dead individuals, they were immediately removed from the dishes. The numbers of dead individuals and presoldier formation (see below) were checked every 24 hours.

We measured the lengths of mandibular apical regions (lengths between apex and first teeth) of white individuals at the end of experiments. Presoldiers (also called white soldiers) are unpigmented and unsclerotized until the next molt to form fully sclerotized soldiers (Lainé and Wright, 2003). In the natural colony, presoldiers and newly molted workers can easily be distinguished by the shape of the mandible; however, individuals that show various mandibular lengths were observed in the JH III treatments (see Results). We could not accurately discriminate newly molted workers and differentiated presoldiers with relatively short mandibles. Thus, there is a possibility that presoldiers induced by JH III include not only presoldiers, but also workers that have recently molted from workers (before pigmentation) (see Results for details). Statistical analysis was performed by the usual method of multiple comparison (Tukey's multiple comparison test) using statistical software, Mac Statistical Analysis ver. 1.5 (Esumi, Tokyo, Japan).

Experiments on the influence of soldiers. Filter papers were treated with 40,80 and $160 \mu \mathrm{g} \mathrm{JH}$ III dissolved in $200 \mu \mathrm{l}$ acetone. Using the methods described above, 20 worker individuals were exposed to each filter paper with $0(0 \%), 1(\sim 5 \%)$, $5(20 \%)$ and $13(\sim 40 \%)$ soldiers. Each treatment was replicated five times. All dishes were kept in an incubator at $23^{\circ} \mathrm{C}$ in constant darkness for 24 days. If the soldiers died, different live soldiers kept in the incubator were added to the dishes. The numbers of dead individuals and presoldier formation (see below) were checked every 24 hours. At the end of experiments, we measured the lengths of mandibular apical regions of presoldiers. Statistical analysis was performed by Tukey's multiple comparison test.

\section{RESULTS}

\section{Experiments on various concentrations of $\mathrm{JH}$ III}

The highest numbers of dead individuals were observed in the dose treated with $320 \mu \mathrm{g}$ JH III in $200 \mu$ acetone $(3.2 \pm 1.3$ [average \pm standard deviations] per 1 dish); however, these numbers were not significantly different from treatments with DW 


\section{a. worker}
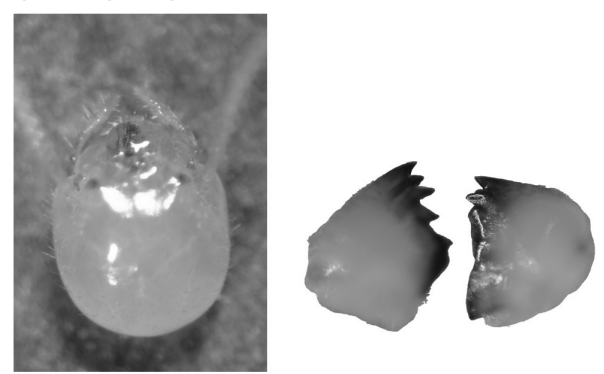

b.
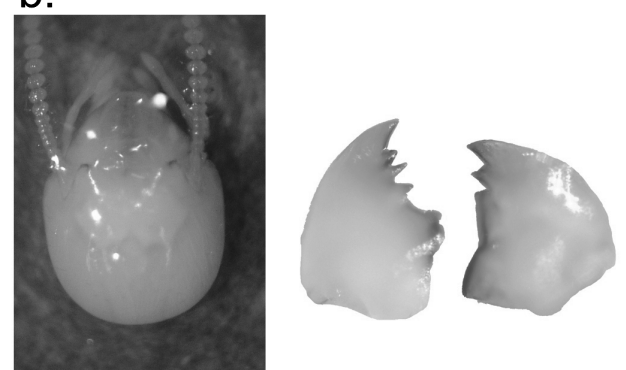

C.
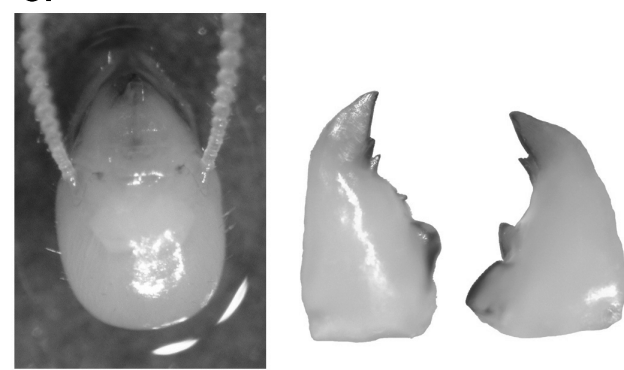

d.
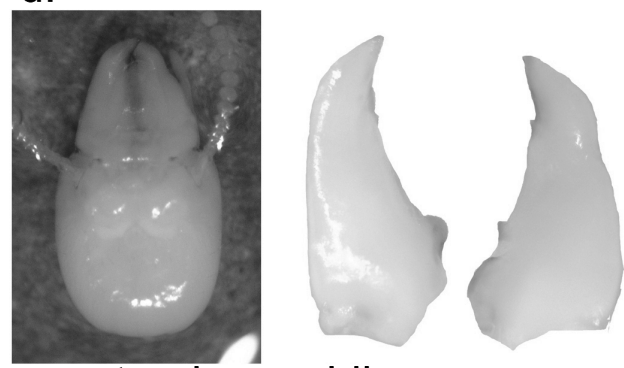

e. natural presoldier
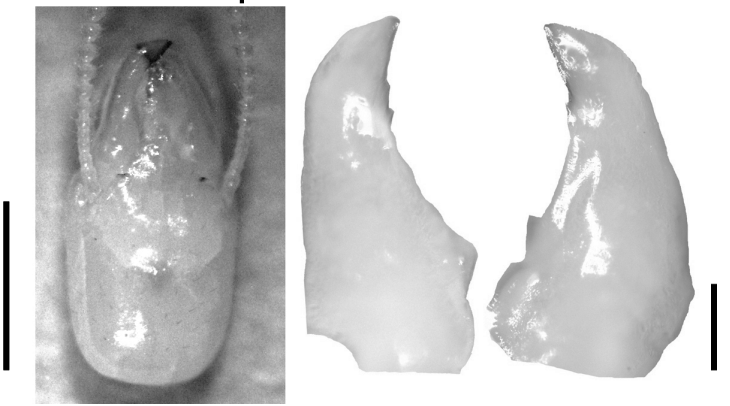

Fig. 1. Head and mandibles of (a) worker (W5; Takematsu, 1992), (b-d) presoldiers induced by JH III, and (e) natural presoldier. Scale bars indicate $1 \mathrm{~mm}$ (head) and $200 \mu \mathrm{m}$ (mandible), respectively. and acetone (both $1.6 \pm 1.1$ per dish, Tukey's multiple comparison test, $p>0.05$ ).

We checked presoldier formation every 24 hours, and found that newly molted white individuals had various-length mandibles (Fig. 1). Figure 2 shows the numbers of these white individuals, and their average lengths of mandibular apical regions (lengths between apex and first teeth) in each dose. Mandibular lengths of those treated with more than $20 \mu \mathrm{g} \mathrm{JH}$ III were significantly longer than those treated with less than $40 \mu \mathrm{g} \mathrm{JH}$ III (including acetone and DW) (Tukey's multiple comparison test, $p<0.05$ ). Presoldier differentiation under treatment with acetone or DW (about 2-3 weeks) is normally very rare in Reticulitermes species (Okot-Kotber et al., 1991; Scharf et al., 2003). Thus, all white individuals receiving less than $40 \mu \mathrm{g} \mathrm{JH}$ III are suggested to be newly molted workers. When treated with more than $20 \mu \mathrm{g} \mathrm{JH}$ III, many differentiated presoldiers (as well as a small number of newly molted workers) were observed. Moreover, concentration-dependent effects on mandibular lengths were observed; higher JH III concentrations caused differentiation of presoldiers with longer mandibles. Although we could find only three natural presoldiers in the experimental colony, the lengths of both left and right mandibles were not significantly different from those treated with 40 , 80,160 and $320 \mu \mathrm{g} \mathrm{JH}$ III $(p>0.05)$.

\section{Experiments on the influence of soldiers}

The numbers of dead worker individuals were not significantly different among treatments at each concentration of JH III [average number: 1.3 $(40 \mu \mathrm{g}), 2.2(80 \mu \mathrm{g})$ and $3.8(160 \mu \mathrm{g}) ; p>0.05]$.

Figure 3 shows the rate of induced presoldiers at the end of the experiments. When treated with $40 \mu \mathrm{g} \mathrm{JH}$ III, the rates of induced presoldiers were relatively low in all treatments and there were no significant differences $(p>0.05)$. When treated with $80 \mu \mathrm{g}$ JH III, however, there were significant differences in the rates of induced presoldiers between treatments with 0,1 and 13 soldiers $(p<$ 0.05 ). When treated with $160 \mu \mathrm{g} \mathrm{JH} \mathrm{III,} \mathrm{there} \mathrm{were}$ no significant differences among treatments, although the highest presoldier-induced rates were observed with 1 soldier.

The numbers of presoldiers and the average length of mandibular apical regions (length between apex and first teeth) in each dose are shown 


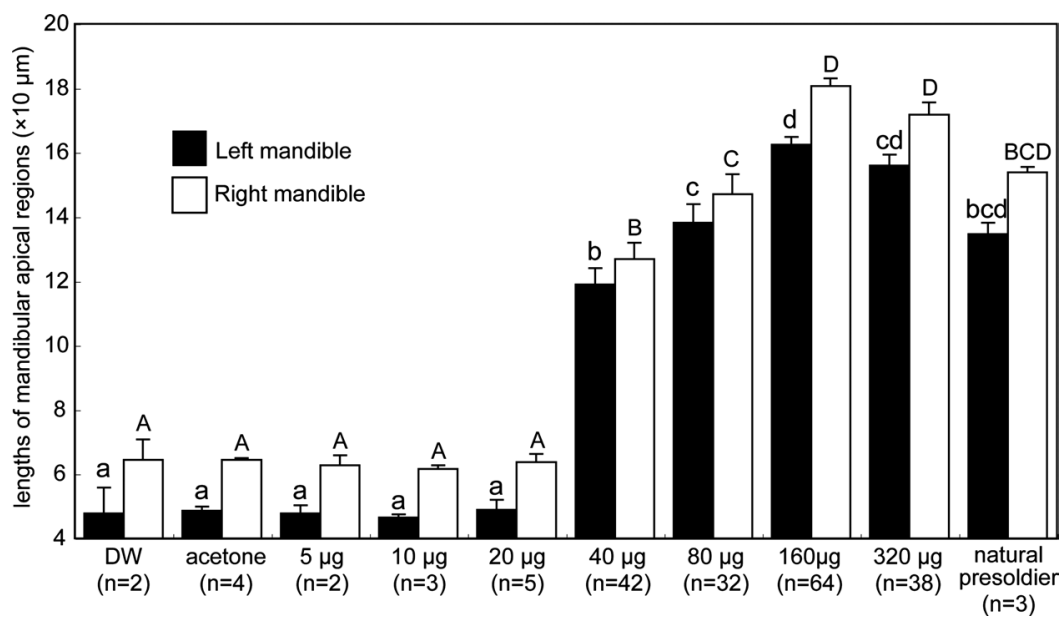

Fig. 2. Average lengths of left (black columns) and right (white columns) mandibular apical regions of white individuals at each dose (numbers of individuals are in parentheses). All white individuals receiving less than $20 \mu \mathrm{g}$ JH III were suggested to be newly molted workers, whereas, when treated with more than $40 \mu \mathrm{g} \mathrm{JH}$ III, presoldiers were observed (see text for the detail). Bars represent standard deviations (SD). Means with different letters were significantly different at $p<0.05$ (Tukey's multiple comparison test).

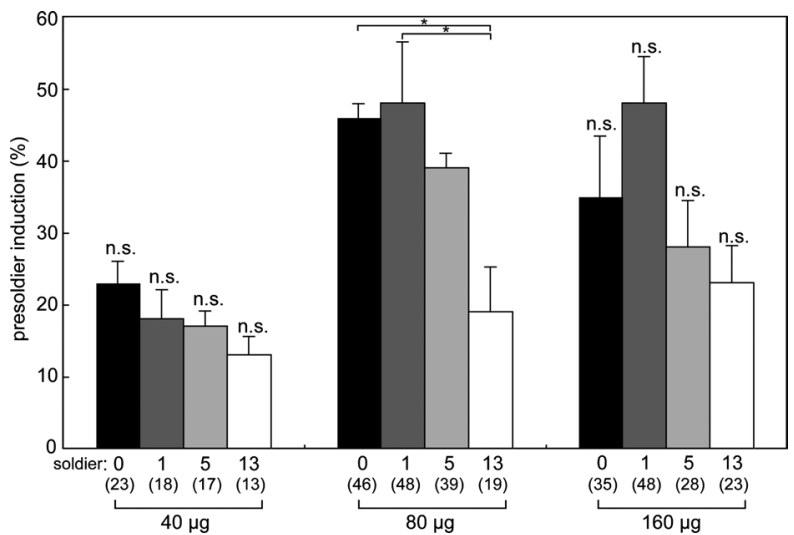

Fig. 3. Average presoldier induction rate per dish in each JH III concentration with live soldiers $(0,1,5$ and 13$)$. Total numbers of individuals are shown in parentheses. Bars represent standard deviations (SD). Asterisk indicates a significant difference in Tukey's multiple comparison test $(p<0.05)$.

in Fig. 4. Both left and right mandibular lengths of presoldiers were clearly short when treated with $40 \mu \mathrm{g} \mathrm{JH}$ III. At this dose, there were no significant differences in mandibular lengths among those treated with different numbers of soldiers $(p>$ 0.05 ), whereas when treated with $80 \mu \mathrm{g} \mathrm{JH}$ III, the longest mandibular lengths were significantly shown when workers had no contact with soldiers $(p<0.05)$. Although similar tendencies were observed when treated with $160 \mu \mathrm{g} \mathrm{JH}$ III, there was only a significant difference between treatments with 0 and 13 soldiers $(p<0.05)$.
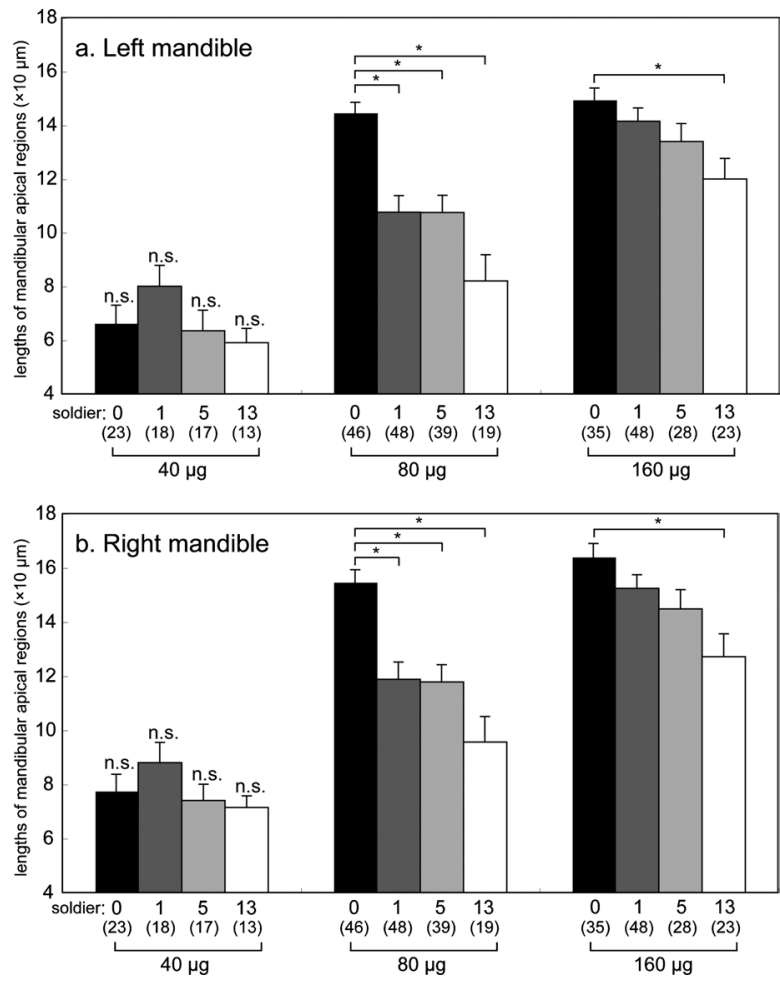

Fig. 4. Average lengths of (a) left and (b) right mandibular apical regions of induced presoldiers in each assay with live soldiers $(0,1,5$ and 13). Numbers of presoldiers in each assay are shown in parentheses. Bars represent standard deviations (SD). Asterisk indicates a significant difference in Tukey's multiple comparison test $(p<0.05)$. 


\section{DISCUSSION}

\section{JH titer and mandibular morphological changes}

This study showed that JH III was effective at inducing presoldier differentiation from $R$. speratus workers, as previously shown in other Reticulitermes species (Scharf et al., 2003); however, presoldiers induced by JH III $(40-320 \mu \mathrm{g})$ had variouslength mandibles (Fig. 1) and concentration-dependent effects on lengths were observed (Fig. 2). Elongated mandibles are a soldier-specific characteristic in lower termites (Lelis and Everaerts, 1993; Koshikawa et al., 2002). The present results suggest that morphogenetic changes specific to soldiers are influenced by JH titers in workers.

Responses to $\mathrm{JH}$ and/or JHA treatments were shown to be very different among colonies of the same species in Reticulitermes, probably because of differences in the physiological stage of the colony and environmental factors (Hrdý et al., 2006). Workers in a colony, even if the same instar, could also show differences in responses to $\mathrm{JH}$ and/or JHA treatments. Although JH titers of $R$. speratus workers have not been investigated, OkotKotber et al. (1993) measured changes in JH titers of $R$. flavipes workers and showed that rapid changes occurred after collection from the field. Park and Raina (2005) also showed changes in JH titers of $C$. formosanus workers; JH titers of workers kept in the laboratory gradually increased. These results suggest that, even in same-instar workers, endogenous JH titers vary among individuals. JH titers of workers after application of JH III in this study could also be variable. Thus, the present results (Fig. 2) suggest that higher JH titers (endogenous $\mathrm{JH}+$ applied JH III) cause differentiation of presoldiers with longer mandibles, and lower JH titers cause differentiation of presoldiers with shorter mandibles. We suggest that JH titers before molting to presoldiers are important for presoldier mandibular elongation and morphogenesis, and that mandibular lengths of presoldiers could be reflected by $\mathrm{JH}$ titers in workers. Although the numbers of presoldiers in the termite colony vary depending on the season, they are not so common (only three individuals were found in this study). Thus, it is not clear whether presoldiers with various-length mandibles exist under natural conditions. We should continue to analyze whether natural presoldiers with significant longer (or shorter) mandibles exist in the same colony. Preliminary histological analyses on the corpora allata (CA) showed that CA sizes (area of maximum cross section; Itano and Maekawa, 2008) of presoldiers induced by JH III $(60 \mu \mathrm{g}$ per dish) were much smaller than those of natural presoldiers (Tsuchiya and Maekawa, unpublished data). Thus, active endogenous JH synthesis is not necessary for workers to molt into presoldiers, if sufficient JH III is applied to workers.

Intercastes between workers and soldiers or mandibular abnormalities were reported under the influence of JH analogues (methoprene, pyriproxyfen and hydroprene) and other $\mathrm{JH}$ homologs ( $\mathrm{JH}$ II) in $R$. santonensis (Lelis and Everaerts, 1993), $R$. flavipes and $R$. tibialis (Scharf et al., 2003); however, JH III treatment of $R$. flavipes and $R$. tibialis workers led to normal morphological features in presoldiers (Scharf et al., 2003). Reticulitermes speratus workers were shown to differentiate to presoldiers, with elongated mandibles, which were not significantly different from those of natural presoldiers, by the application of JH III (40-320 $\mu \mathrm{g}$ per dish; Fig. 2). Positively correlated relationships were also shown between JH III concentrations in workers and mandibular lengths of induced presoldiers (Fig. 2). This is the first report identifying continuous changes of soldier-specific characteristics during presoldier differentiation induced by $\mathrm{JH}$ itself (not JHA nor JH I/II). Termite caste differentiation is a fine example of insect polyphenism, and the phenomenon has relatively discrete or discontinuous variation in phenotypic expression (Nijhout, 1994; Hartfelder and Emlen, 2005). Soldier differentiation is normally accompanied with irreversible morphological changes, and complex epithelial configuration was observed during mandibular morphogenesis prior to presoldiers in Hodotermopsis sjostedti (Koshikawa et al., 2002). We suggest that these mandibular morphogenetic events are under the control of JH titers, and that high epidermal growth rates and/or speed are caused by high JH titers in workers. Further analyses are needed to determine whether other morphological changes correlated with $\mathrm{JH}$ titers occurred during presoldier differentiation (for example, frontal-gland morphogenesis). Moreover, we were not able to determine the mandibular morphology of soldiers, which molted from induced presoldiers in this study. Presoldiers always molt 
into soldiers, not into workers. Worker-presoldier molts are thus extremely important for the investigation of caste differentiation associated with soldier-specific morphological changes. Consequently, in this study, we concentrated on analyses of the morphological changes from workers to presoldiers. Further analyses are needed to identify morphological differences among soldiers induced by different concentrations of JH III.

\section{Presence of soldiers and mandibular morpho- logical changes}

As previously shown in closely related species $[R$. flavipes (Okot-Kotber et al., 1991) and $C$. formosanus (Park and Raina, 2003)], as well as in distantly related termites [Nasutitermes lujae (Lefeuve and Bordereau (1984)], live soldiers could inhibit presoldier differentiation from $R$. speratus workers treated with JH III $(80 \mu \mathrm{g}$ per dish; Fig. 3). Preliminary analyses showed that, when the initial numbers of workers were changed (20 and 70 individuals per dish), the rates of presoldier differentiation treated with sufficient $\mathrm{JH}$ III concentrations were not different (about 40 $50 \%$ ). Soldier presence, thus, could be essentially important for the inhibition of presoldier differentiation. The present results suggest that inhibiting effects are strongly related to the numbers of living soldiers. When workers were treated with JH III (40 and $160 \mu \mathrm{g}$ per dish), the rates of induced presoldiers were not significantly different among treatments. The application of $40 \mu \mathrm{g} \mathrm{JH}$ III did not strongly induce presoldier differentiation, thus, differences in induced-presoldier rates could not be detected among treatments with soldiers. Meanwhile, the application of $160 \mu \mathrm{g}$ JH III showed stronger effects of presoldier induction, thus, it was shown that there were no significant differences among treatments. The rates of induced presoldiers were lower for high initial soldier proportion treatments, and no effects of inhibition were shown when workers were exposed to one soldier. A higher proportion of soldiers was suggested to be necessary to inhibit further presoldier formation in C. formosanus (Park and Raina, 2003). Moreover, JH titers in workers were higher in low initial soldier proportions (Mao et al., 2005; Park and Raina, 2005). The present results also suggest that soldier proportions regulate $\mathrm{JH}$ titers in workers and presoldier differentiation in $R$. speratus, as shown in C. formosanus.

Most importantly and interestingly, the present study showed that mandibular lengths of presoldiers induced by JH III were influenced by soldier presence (Fig. 4), which could influence morphological changes, probably by the regulation of $\mathrm{JH}$ titers in workers (Mao et al., 2005; Park and Raina, 2005). Figure 5 shows the proposed model between mandibular lengths and $\mathrm{JH}$ titers, suggesting that there could be a threshold for JH titers for differentiation from workers to presoldiers (Yin and Gillott, 1975; Hartfelder and Emlen, 2005). If total $\mathrm{JH}$ titers (endogenous JH+applied JH III) in workers increase and exceed the threshold, presoldier differentiation and high mandibular epidermal growth could occur (Fig. 5a). We suggest that mandibular lengths are influenced by JH titers: higher JH titers cause differentiation of presoldiers

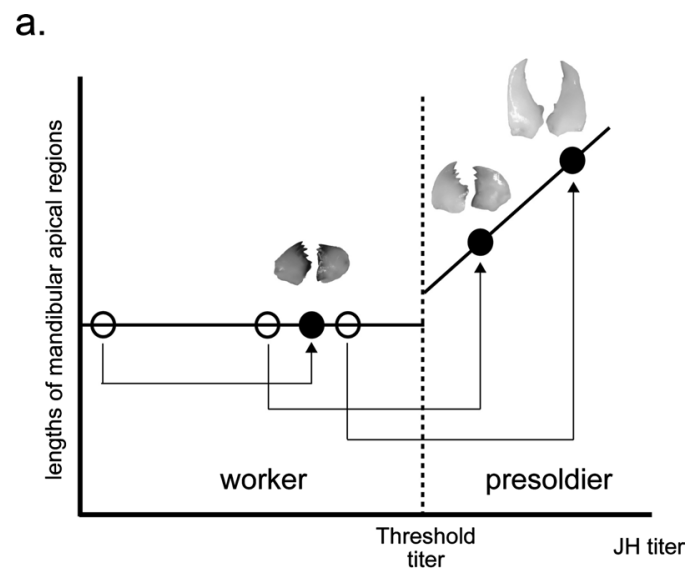

b.

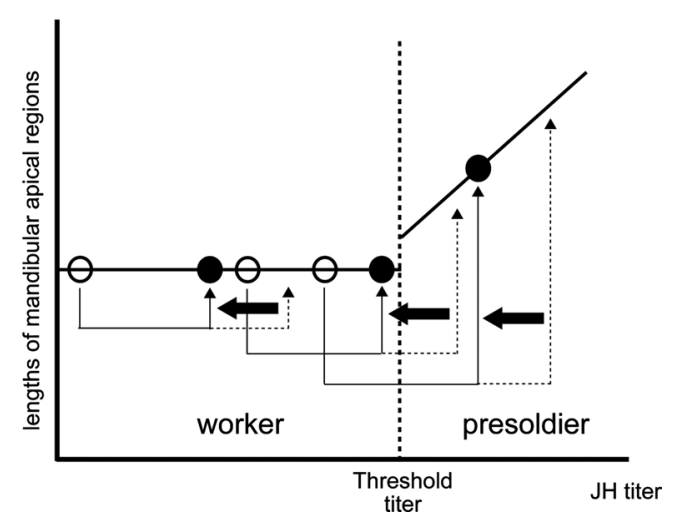

Fig. 5. Proposed schemata of the relationships between $\mathrm{JH}$ titers in workers and lengths of mandibular apical regions. (a) JH III treatment, and (b) effects of soldier presence. White and black dots indicate individuals before and after treatments, respectively. Soldiers probably bring about the reduction of $\mathrm{JH}$ titers in workers (b, arrow). 
with longer mandibles, and lower $\mathrm{JH}$ titers cause differentiation of presoldiers with shorter mandibles. When soldiers were present, the numbers of differentiated presoldiers (especially having longer mandibles) decreased, probably because of the reduction of JH titers in workers (Mao et al., 2005; Park and Raina, 2005) (Fig. 5b). Preliminary histological analyses on the CA of induced presoldiers in the presence of soldiers showed that their CA sizes were significantly larger than those of natural presoldiers (Tsuchiya and Maekawa, unpublished data). These results suggest that soldier presence probably influences $\mathrm{JH}$ metabolism (sequestration or degradation) after their synthesis from CA. Because $\mathrm{JH}$ treatments might potentially affect the release of other factors related to the alterations of phenotypic expression (reviewed in Zera, 2007), detailed direct measurements of variation in $\mathrm{JH}$ titers and regulators (especially $\mathrm{JH}$-binding protein; Zhou et al., 2006) are needed. Moreover, to understand common and/or different effects on workers by soldier presence, further analyses on other characteristics (e.g. frontal-gland and reproductiveorgan morphogenesis) during presoldier differentiation are needed. We are now expanding our study to address these issues.

\section{ACKNOWLEDGEMENTS}

We thank Drs. Toru Miura, Shigeyuki Koshikawa, Richard Cornette, Tadao Matsumoto and Kunio Suzuki who gave us valuable comments on the manuscript. Thanks are also due to Sayaka Mizuno, Satoshi Nakata, Yusuke Nakamura, Keisuke Shimada, Yoshinaka Urasoko, and Kana Yoshihira for their help during our field and laboratory work. This study was partly supported by a Grant-in-Aid for Scientific Research (Nos. 16770014 and 19770012 to KM) from the Japan Society for the Promotion of Science.

\section{REFERENCES}

Doki, H., K. Tsunoda and K. Nishimoto (1984) Effect of juvenile hormone analogues on caste-differentiation of the termite, Reticulitermes speratus (Kolbe) (Isoptera: Rhinotermitidae). Material u. Organismen 19: 175187.

Hartfelder, K. (2000) Insect juvenile hormone: from "status quo" to high society. Braz. J. Med. Biol. Res. 33: 157177.

Hartfelder, K. and D. J. Emlen (2005) Endocrine control of insect polyphenism. In Comprehensive Molecular Insect Science. Vol. 3 (L. I. Gilbert, K. Iatrou and S. S. Gill eds.). Elsevier, Amsterdam, pp. 651-703.

Haverty, M. I. (1977) The proportion of soldiers in termite colonies: a list and a bibliography. Sociobiology 2: 199-
216.

Hrdý, I., J. Kuldová, R. Hanus and Z. Wimmer (2006) Juvenile hormone III, hydroprene and a juvenogen as soldier caste differentiation regulators in three Reticulitermes species: potential of juvenile hormone analogues in termite control. Pest Manag. Sci. 62: 848-854.

Itano, H. and K. Maekawa (2008) Soldier differentiation and larval juvenile hormone sensitivity in an incipient colony of the damp-wood termite Zootermopsis nevadensis (Isoptera, Termopsidae). Sociobiology 51: 151-162.

Koshikawa, S., T. Matsumoto and T. Miura (2002) Morphometric changes during soldier differentiation of the dampwood termite Hodotermopsis japonica (Isoptera: Termopsidae). Insect. Soc. 49: 245-250.

Lainé, L. V. and D. J. Wright (2003) The life cycle of Reticulitermes spp. (Isoptera: Rhinotermitidae): what do we know? Bull. Entomol. Res. 93: 267-278.

Lefeuve, P. and C. Bordereau (1984) Soldier formation regulated by a primer pheromone from the soldier frontal gland in a higher termite, Nasutitermes lujae. Proc. Natl. Acad. Sci. USA 81: 7665-7668.

Lelis, A. T. and C. Everaerts (1993) Effects of juvenile hormone analogues upon soldier differentiation in the termite Reticulitermes santonensis (Rhinotermitidae, Heterotermitinae). J. Morphol. 217: 239-261.

Mao, L., G. Henderson, Y. Liu and R. A. Laine (2005) Formosan subterranean termite (Isoptera: Rhinotermitidae) soldiers regulate juvenile hormone levels and caste differentiation in workers. Ann. Entomol. Soc. Am. 98: 340345.

Nijhout, H. F. (1994) Insect Hormones. Princeton University Press, Princeton. 267 pp.

Ogino, K., Y. Hirono, T. Matsumoto and H. Ishikawa (1993) Juvenile hormone analogue, S-31183, causes a high level induction of presoldier differentiation in the Japanese damp-wood termite. Zool. Sci. 10: 361-366.

Okot-Kotber, B. M., I. Ujvary, R. Mollaaghababa, F. Szurdoki, G. Matolcsy and G. D. Prestwich (1991) Physiological influence of fenoxycarb pro-insecticides and soldier head extracts of various termite species on soldier differentiation in Reticulitermes flavipes (Isoptera). Sociobiology 19: 77-90.

Okot-Kotber, B. M., G. D. Prestwich, A. Strambi and C. Strambi (1993) Changes in morphogenetic hormone titers in isolated workers of the termite Reticulitermes flavipes (Kollar). Gen. Comp. Endocrinol. 90: 290-295.

Park, Y. I. and A. K. Raina (2003) Factors regulating caste differentiation in the Formosan subterranean termite with emphasis on soldier formation. Sociobiology 41: 49-60.

Park, Y. I. and A. K. Raina (2004) Juvenile hormone III titers and regulation of soldier caste in Coptotermes formosanus (Isoptera: Rhinotermitidae). J. Insect Physiol. 50: 561-566.

Park, Y. I. and A. K. Raina (2005) Regulation of juvenile hormone titers by soldiers in the Formosan subterranean termite, Coptotermes formosanus. J. Insect Physiol. 51: 385-391.

Roisin, Y. (2000) Diversity and evolution of caste patterns. In Termites: Evolution, Sociality, Symbioses, Ecology (T. 
Abe, D. E. Bingnell and M. Higashi eds.). Kluwer Academic Publishers, Dordrecht, pp. 95-119.

Scharf, M. E., C. R. Ratliff, J. T. Hoteling, B. R. Pittendrigh and G. W. Bennett (2003) Caste differentiation responses of two sympatric Reticulitermes termite species to juvenile hormone homologs and synthetic juvenoids in two laboratory assays. Insect. Soc. 50: 346-354.

Takematsu, Y. (1992) Biometrical study on the development of the castes in Reticulitermes speratus (Isoptera, Rhinotermitidae). Jpn. J. Ent. 60: 67-76.

Tsunoda, K., H. Doki and K. Nishimoto (1986) Effect of developmental stages of workers and nymphs of Reticulitermes speratus (Kolbe) (Isoptera: Rhinotermitidae) on caste differentiation induced by JHA treatment. Material $u$. Organismen 21: 47-61.
Weesner, F. M. (1969) External anatomy. In Biology of Termites. Vol. I (K. Krishna and F. M. Weesner eds.). Academic Press, New York, pp. 19-47.

Yin, C. M. and C. Gillot (1975) Endocrine control of castedifferentiation in Zootermopsis angusticollis Hagen (Isoptera). Can. J. Zool. 53: 1701-1708.

Zera, A. J. (2007) Endocrine analysis in evolutionary-developmental studies of insect polymorphism: hormone manipulation versus direct measurement of hormonal regulators. Evol. Dev. 9: 499-513.

Zhou, X., F. M. Oi and M. E. Scharf (2006) Social exploitation of hexamerin: RNAi reveals a major caste-regulatory factor in termites. Proc. Natl. Acad. Sci. USA 103: 4499-4504. 